\title{
Commands 4 Autonomous Vehicles (C4AV) Workshop Summary
}

\author{
(2) Thierry Deruyttere ${ }^{1, \star} \quad$ Simon Vandenhende ${ }^{2, \star} \quad$ Dusan Grujicic $^{2, \star} \quad \mathrm{Yu} \mathrm{Liu}^{2}$

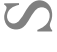 \\ Luc Van Gool $^{2}$ Matthew Blaschko ${ }^{2}$ Tinne Tuytelaars ${ }^{2}$ \\ Marie-Francine Moens ${ }^{1}$ \\ KU Leuven \\ ${ }^{1}$ Department of Computer Science (CS) \\ ${ }^{2}$ Department of Electrical Engineering (ESAT) \\ ${ }^{1}$ \{thierry.deruyttere, sien.moens\}@cs.kuleuven. be \\ ${ }^{2}$ f firstname.lastname\}@esat . kuleuven. be
}

\begin{abstract}
The task of visual grounding requires locating the most relevant region or object in an image, given a natural language query. So far, progress on this task was mostly measured on curated datasets, which are not always representative of human spoken language. In this work, we deviate from recent, popular task settings and consider the problem under an autonomous vehicle scenario. In particular, we consider a situation where passengers can give free-form natural language commands to a vehicle which can be associated with an object in the street scene. To stimulate research on this topic, we have organized the Commands for Autonomous Vehicles (C4AV) challenge based on the recent Talk2Car dataset. This paper presents the results of the challenge. First, we compare the used benchmark against existing datasets for visual grounding. Second, we identify the aspects that render top-performing models successful, and relate them to existing state-of-the-art models for visual grounding, in addition to detecting potential failure cases by evaluating on carefully selected subsets. Finally, we discuss several possibilities for future work.
\end{abstract}

\section{Introduction}

The joint understanding of language and vision poses a fundamental challenge for the development of intelligent machines. To address this problem, researchers have studied various related topics such as visual question answering [3, 1, 19, 40, 17, image-captioning [49, 51, visual grounding [9, 53, 34, etc. These advancements can provide a stepping stone towards new products and services. For example, a natural language interface between factory operators and control systems could streamline production processes, resulting in safer and more efficient working environments. In a different vein, providing passengers with the possibility to communicate with their autonomous car could eliminate the unsettling feeling of giving up all control. The possible applications are countless.

\footnotetext{
* Authors contributed equally.
} 
Understandably, this calls for efficient computational models that can address these tasks in a realistic environment.

In this paper, we focus on the task of visual grounding. Under this setup, the model is tasked with locating the most relevant object or region in an image based on a given natural language query. Several approaches [9, 53, 34, 50, 16, 20. tackled the problem using a two-stage pipeline, where region proposals are generated first by an off-the-shelf object detector [33, 31, and then matched with an embedding of the sentence. Others [18, 14] proposed an end-to-end strategy where the object location is predicted directly from the input image.

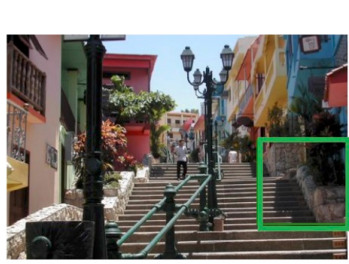

(a) ReferIt

right rocks

rocks along the right side

stone right side of stairs

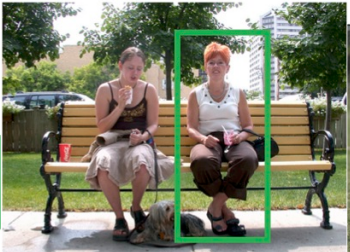

(b) RefCOCO

woman in white shirt woman on right right woman

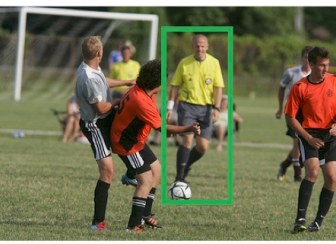

(c) RefCOCO+ guy in yellow dribbling ball yellow shirt black shorts yellow shirt in focus

Fig. 1: Examples from popular benchmarks for visual grounding [21].

In order to quantify progress, several benchmarks were introduced [21, 54, 27, 15. From visualizing examples found in existing datasets in Figure 1, we draw the following conclusions. First, we observe that the language queries are rather artificial, and do not accurately reflect the type of language used by human speakers during their daily routines. For example, in practice, object references are often implicitly defined, complex and long sentences can contain co-referent phrases, etc. Second, existing benchmarks are mostly built on web-based image datasets [24, 13, 52, where the object of interest is often clearly visible due to its discriminative visual features. From these observations, we conclude that existing benchmarks for visual grounding are not well suited to develop models that need to operate in the wild.

To address these shortcomings, we hosted the Commands For Autonomous Vehicles Challenge (C4AV) at the European Conference on Computer Vision (ECCV) 20'. The challenge setting considers a visual grounding task under a self-driving car scenario. More specifically, a passenger gives a natural language command to express an action that needs to be taken by the autonomous vehicle $(\mathrm{AV})$. The model is tasked with visually grounding the object that the command is referring to. The recently proposed Talk2Car dataset [10] is used to run the challenge. Some examples are displayed in Figure2 2 An extensive description of the challenge can be found in Section 3 . 
In contrast to existing benchmarks, several additional challenges are encountered on the Talk2Car dataset. First, the referred object can be ambiguous (e.g. there are multiple pedestrians in the scene), but can be disambiguated by understanding modifier expressions in language (e.g. the pedestrian wearing the blue shirt). In some cases, the modifier expressions also indicate spatial information. Second, detecting the correct object is challenging both in the language utterance and the urban scene, for example, when dealing with long and complex sentences, and with small objects in the visual scene, respectively. Finally, the model size and the execution time also play an important role under the proposed task setting.

The contributions of our work are as follows:

- We propose the first challenge for grounding commands for self-driving cars in free natural language into the visual context of an urban environment.

- We scrutinize the results obtained by top performing teams. In particular, we compare them against several well-known state-of-the-art models, and further evaluate them on carefully selected subsets that address different key aspects of solving the task at hand, in order to identify potential failure cases.

- Finally, we identify several possibilities for future work under the proposed task setting.

\section{Related Work}

This section provides an overview of recent work on visual grounding. First, several methods are discussed, including both region proposal and non-region proposal based strategies. Next, we review existing benchmarks for visual grounding.

\section{$2.1 \quad$ Methods}

Existing solutions for visual grounding can be subdivided into two main groups of works. In Region Proposal Based methods, object proposals are first generated for the image using an off-the-shelf object detector (typically the region proposal network - RPN). Different works have considered how to correctly match the extracted regions with the language query. In Non-Region Proposal Based methods, a model reasons over the full image directly, instead of first extracting object proposals. We discuss representative works for both groups next.

Region Proposal Network (RPN) Based Methods Hu et al. [16] train a model to maximize the likelihood of the referring expression for region proposals that match the object of interest. The global context, spatial configuration and local image features are all taken into account. Rohrbach et al. 34 tackle the problem by learning to attend to regions in the image from which the referring expression can be reconstructed. To this end, they serve a visual representation of the attended regions as input to a text-generating RNN. Wang et al. [50] 
learn a joint embedding for image regions and expressions by enforcing proximity between corresponding pairs through a maximum-margin ranking loss. More recently, modular approaches have seen an increase in popularity. For example, MAttNet 53] decomposes the referring expression into three distinct components, i.e. subject appearance, location and spatial relationships. The different components are subsequently matched with the visual representations, and combined to get a score for each region in the image. Similarly, MSRR 9] uses separate modules that focus on text, image and spatial location, and ranking of the image regions respectively. Additionally, the predictions of each module are improved in a recursive manner.

Non-Region Proposal Based Methods Hu et al. [15] apply a modular approach directly to the input image. First, they develop a set of modules that each execute a specific task, and return an attention map over image regions. Next, the expression is decomposed into sub-parts using attention. The extracted parts are considered as sub-problems that can be tackled by the smaller modules learned during the first step. Finally, the answers of the different sub-modules are integrated through an attention mechanism. In contrast to region proposal based methods, the image is subdivided into a 2-dimensional grid. The model predicts the grid cell containing the center of the referred object together with the bounding box offset.

Another approach that does not rely on the use of region proposals is the work of Hudson and Manning 18. Although this method was originally developed for visual question answering, [10] adapted it to tackle the visual grounding task. The model uses a recurrent $M A C$ cell to match the natural language command with a global representation of the image. First, the MAC cell decomposes the textual input into a series of reasoning steps. Additionally, the MAC cell uses the decomposed textual input to guide the model to focus on certain parts in the image. Information is passed to the next cell between each of the reasoning steps, allowing the model to represent arbitrarily complex reasoning graphs in a soft sequential manner.

\section{$2.2 \quad$ Datasets}

A number of datasets have been proposed to benchmark progress on the visual grounding task. These include both real-world [21, 54, 27] and synthetic [14] datasets. An overview is provided in Table 1. Some of the most commonly used datasets are ReferIt [21, RefCOCO [54, RefCOCO+ [54] and RefCOCOg [27. These datasets were constructed by adding textual annotations on top of the well-known MS COCO dataset 24]. Examples of image-sentence pairs sampled from these datasets can be seen in Figure 1. Notice that the language utterances are rather artificial, i.e. the queries do not accurately present the language used by human speakers. On the other hand, the examples in Figure 2 feature expressions that are more representative of everyday language, e.g. object references are often less explicit, and part of longer and more complex sentences. Additionally, the images found in the aforementioned datasets were collected from the 
web, and as a consequence, the objects of interest are often quite easy to spot. A different situation arises when considering indoor or urban scene environments.

In contrast to prior works, Vasudevan et al. 47] and Deruyttere et al. 10] considered the visual grounding task in a city environment. The main difference between the two works is the use of object descriptions in the former, versus the use of command-like expressions with more implicit object references in the latter. In this work, we use the Talk2Car [10] dataset.

\begin{tabular}{|l|c|c|c|c|c|c|c|}
\hline Dataset & Images & Objects & Expressions & Avg expr length & Video & Lidar & Radar \\
\hline ReferIt [21] & 19,894 & 96,654 & 130,525 & 3.46 & $\times$ & $\times$ & $\times$ \\
RefCOCO [54] & 26,711 & 50,000 & 142,209 & 3.61 & $\times$ & $\times$ & $\times$ \\
RefCOCO+ [54] & 19,992 & 49,856 & 141,564 & 3.53 & $\times$ & $\times$ & $\times$ \\
RefCOCOg [27] & 26,711 & 54,822 & 85,474 & 8.43 & $\times$ & $\times$ & $\times$ \\
CLEVR-Ref [14] & 99,992 & 492,727 & 998,743 & 14.50 & $\times$ & $\times$ & $\times$ \\
Cityscapes-Ref [47]] & 4,818 & 29,901 & 30,000 & 15.59 & $\checkmark$ & $\times$ & $\times$ \\
Talk2Car [10] & 9,217 & 10,519 & 11,959 & 11.01 & $\checkmark$ & $\checkmark$ & $\checkmark$ \\
\hline
\end{tabular}

Table 1: An overview of public datasets for visual grounding [10.

\section{Commands for Autonomous Vehicles Challenge}

This section describes the 'Commands for Autonomous Vehicles' (C4AV) challenge that was hosted as part of the C4AV workshop at ECCV 20'. First, we introduce the used benchmark. Second, we define three baseline models that were provided at the start of the challenge. Finally, we give an overview of the top performing models at the end of the competition.

\subsection{Dataset}

The C4AV challenge is based on the Talk2Car dataset [10]. The dataset is built on top of the nuScenes [4 dataset which contains 3D object boxes, videos, lidar and radar data obtained by driving a car through Boston and Singapore. Furthermore, the nuScenes dataset covers various weather conditions, different lighting conditions (day and night), and driving directions (left and right). The Talk2Car dataset is constructed by adding textual annotations on top of the images sampled from the nuScenes training dataset. More specifically, the text queries consist of commands provided by a passenger to the autonomous vehicle. Each command can be associated with an object visible in the scene. Figure 2 shows text-image pairs from the Talk2Car dataset. The challenge required to predict the $2 \mathrm{D}$ bounding box coordinates around the object of interest. Additionally, for every image, we provided pre-computed region proposals extracted with a CenterNet [55] model. 


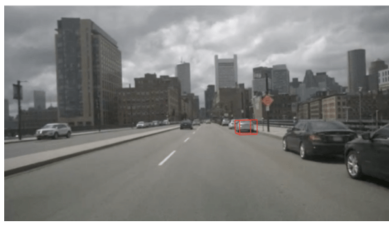

(a) You can park up ahead behind the silver car, next to that lamppost with the orange sign on it

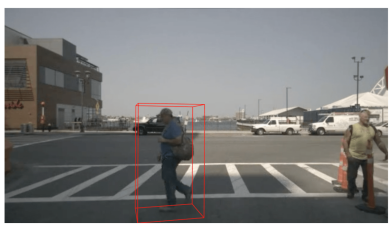

(d) After that man in the blue top has passed, turn left

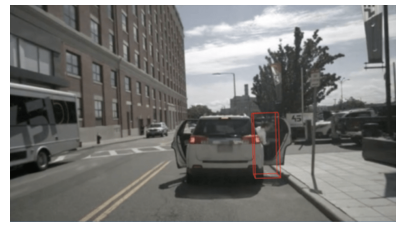

(b) My friend is getting out of the car. That means we arrived at our destination! Stop and let me out too!



(e) There's my mum, on the right! The one walking closest to us. Park near her, she might want a lift

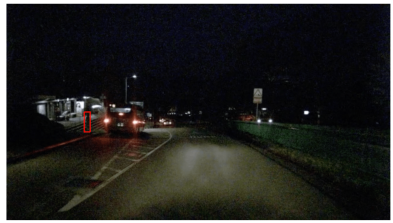

(c) Yeah that would be my son on the stairs next to the bus. Pick him up please

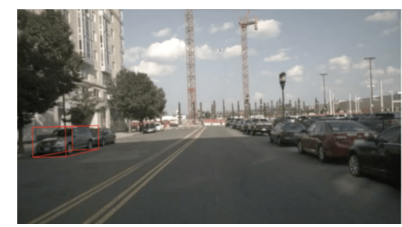

(f) Turn around and park in front of that vehicle in the shade

Fig. 2: Some examples from the Talk2Car dataset [10]. Each command describes an action that the car has to execute relevant to a referred object found in the scene (here indicated by the red 3D-bounding box). The referred object is indicated in bold in each command. Best seen in color.

The dataset contains 11959 text-image pairs in total. The train, val and test set contain $70 \%, 10 \%$ and $20 \%$ of the samples, respectively. Additionally, the test set can be subdivided into four sub-sets of increasing difficulty. One subset was created to study the specific case of objects that are far away from the vehicle. Two sub-sets contain varying command lengths. The fourth subset tests how well the model disambiguates between objects of the same class as the target.

The C4AV challenge was hosted on AICrowd prior to ECCV 20'. Every team was allowed a maximum of three submissions per day. The submissions were evaluated on the held-out test set from the Talk2Car dataset. The evaluation criterion is described in Section 4. Top performing teams were invited to submit a paper to the workshop after undergoing a code verification phase.

\subsection{Baselines}

The leader board featured three baseline models at the start of the challenge. We describe each of them below. For brevity, we adopt the following notations. 
Each model takes as input an image $I$ and a natural language expression under the form of a command $C$. The objective is to localize the referred object $o$.

Bi-directional retrieval Similar to the work from Karpathy et al. [20, a Bidirectional retrieval model is considered. First, a pre-trained object detector [55] is used to generate region proposals from the image $I$. Second, a pre-trained ResNet-18 model is used to obtain a local feature representation for the extracted regions. Similarly, the command $C$ is encoded by a bi-directional GRU. Finally, we match the command $C$ with the correct region proposal. To this end, we maximize the inner product between the local image features of the correct region and the text encoding, while we minimize the inner product for the other regions. To help participants get started in the challenge, a PyTorch [30] implementation of the Bi-directional retrieval model was made publicly available [44].

MAC As a second baseline, we consider the MAC model [17], adapted for the visual grounding task by Deruyttere et al. [10. MAC implements a multi-step reasoning approach, and unlike the Bi-directional retrieval model, does not rely on pre-computed region proposals. First, the image is encoded by a pre-trained ResNet model [12, and the command tokens are encoded by a bidirectional LSTM. Second, the model initiates a multi-step reasoning process by applying attention to the command tokens. In each reasoning step, the model attends to a different word, decomposing the language query into smaller sub-problems. Simultaneously, visual information is extracted from the image through a softattention mechanism conditioned on the attended word. The extracted information is stored as a memory vector, and forwarded to the next reasoning step. The final product of the multi-step reasoning process is a $2 \mathrm{D}$ bounding box derived from the soft-attention mask applied to the visual features.

MSRR As a final baseline model, we consider the prior state-of-the-art on the Talk2Car dataset. Similar to MAC [17, the MSRR model [9] employs a multistep reasoning strategy. However, unlike MAC, MSRR obtains its predictions by ranking object region proposals as in Section 3.2. First, each region is associated with a separate spatial map for which we indicate the spatial location by assigning ones to the regions, while zeros otherwise. Next, MSRR decomposes the expression $C$ into sub-parts through attention and a region dependent reasoning process. The latter is achieved by (i) multiplying the spatial location of each region and its score with the image features, extracted by a ResNet model, and (ii) applying soft-attention conditioned onto the decomposed sub-expression. The result is combined into a region specific memory vector which can be used to score the region based on the alignment of the newly created memory vector and the command $c$. Finally, the highest scoring region is returned. 


\subsection{State-of-the-art models}

Teams that outperformed all three baselines at the end of the challenge were invited to submit a paper to the workshop detailing their solution. A selection of top performing models is summarized below.

Stacked VLBert Dai et al. 8] propose a visual-linguistic BERT model named Stacked VLBert. The approach relies on region proposals, similarly to the MSRR [9] and the Bi-directional retrieval model [44]. Furthermore, the authors propose a weight stacking method to efficiently train a larger model from a shallow VLBert model. The weight stacking procedure is performed by first training a smaller VLBert variant and then copying its trained weights in a repeated manner into a larger model. They show that by doing this, they can achieve a higher score compared to a larger model initialised with random weights. The Stacked VLBert model is conceptually close to the Bi-directional retrieval model as they first encode the image and object regions with a pre-trained image encoder. They then pass the sentence, the encoded image, and the encoded objects to the VLBert model to find the referred object.

Cross-Modal Representations from Transformers (CMRT) Luo et al. [25] also advocate the use of a region proposal based approach. The commands are fed to the input of a transformer encoder, while the image features are used as the query for the transformer decoder. The image features are refined based on the extracted linguistic features obtained from the encoder, which are used as the key and value input to the multi-head attention layers in the decoder. Unlike the common approaches that leverage the transformer encoder alone to extract visual-linguistic features, CMRT uses the transformer decoder to aggregate features from the two modalities. Additionally, as opposed to extracting local features from the region crops, the features of the whole image are used as the decoder input. This allows to capture long-range dependencies in the image, which is important, since the Talk2Car dataset commands also include the surroundings in the description of the objects. After aggregating the feature representations and extracting the feature map at the decoder output, an RoI alignment operation is used to select local image features of interest. The cropped features are fed to a final weight sharing network which is optimized using the same objective function as the Bi-directional retrieval model.

Cosine meets Softmax: A tough-to-beat baseline for visual grounding (CMSVG) Rufus et al. 35] showed that the Bi-directional retrieval approach can outperform more sophisticated approaches such as MSRR [9] and MAC [17] by simply using state-of-the-art object and sentence encoders. They also performed extensive ablation studies to analyse the influence of the used number of region proposals, the used image encoder, and the used text encoder. 


\section{Attention Enhanced Single Stage Multi-Modal Reasoner (ASSMR)} $\mathrm{Ou}$ and Zhang 29] also encode the local information of pre-computed region proposals first. Additionally, the position and scale of the region proposals are encoded and supplied as extra information for every region. Note that these properties are often found in the modifier expressions of the commands, and are thus potentially informative of the object location. Next, the local image features are combined with an encoding of the command to compute the weight for every region, emphasizing the ones most relevant for the given command. Afterwards, the weighted region features are augmented with a global image representation, and aggregated with the hidden states of a GRU that was used to encode the command. An attention mechanism is applied to the obtained multi-modal feature representation and the local object features to compute a final score for each region.

AttnGrounder: Talking to Cars with Attention Mittal 28] proposes a one-stage approach to the visual grounding task. A Darknet-53 31 backbone is utilized for extracting image features at multiple spatial resolution, while a bidirectional LSTM is used to generate text features. A visual-text attention module that relates every word in the given query with different image regions is used to construct a unique text representation for each region. Additionally, the prediction of a segmentation mask within the bounding box of the referred object is introduced as an auxiliary task, improving the localization performance. The predicted mask serves as an attention map used to weigh the visual features, which are subsequently concatenated with the spatially attended text features and the original visual features along the channel dimension, and fused together by using $1 \mathrm{x} 1$ convolutions. Finally, similarly to YOLOv3 [31, the fused features are used to predict the offset from anchor boxes and categorical labels that indicate whether the predicted bounding box corresponds to the ground truth.

\section{Evaluation}

Every model needs to output a 2D bounding box that indicates the location of the referred object. The challenge submissions were ranked using the evaluation measure from Deruyttere et al. [10]. In particular, we employed the $I o U_{.5}$ metric by thresholding the Intersection over Union ( $\mathrm{IoU}$ ) between the predicted and ground-truth bounding boxes at 0.5 . The IoU is defined as follows:

$$
I o U=\frac{\text { Area of Overlap of the two boxes }}{\text { Area of Union of the two boxes }} .
$$

While the challenge focused on the quality of the predictions, other properties such as model size and inference speed are arguably important as well in our task setting. To draw attention to these problems, for every model, we also report the number of parameters and the inference speed on a Nvidia RTX Titan 2080. 


\section{$5 \quad$ Experiments}

This section analyses the results obtained by the models described in Section 3.2 and 3.3. In particular, Section 5.1 draws a comparison between the state-of-theart on the Talk2Car dataset. Section 5.2 evaluates the models on carefully selected subsets to better understand any existing failure cases. Finally, Section 5.3 analyses the commonalities and differences between the used models to isolate the elements that render top-performing models successful.

\subsection{State-Of-The-Art Comparison}

Table 2 compares the models from Section 3.2 and 3.3 on the Talk2Car test set. We compare the models in terms of $I o U_{.5}$, the number of parameters in millions (M) and inference speed in milliseconds (ms). The state-of-the-art prior to the challenge was the MSRR model [9. Notably, the top-performing models from the challenge $[8,25,35,229,28$ show significant gains over the prior state-ofthe-art [9] in terms of performance $\left(I o U_{.5}\right)$. In particular, the Stacked VLBert model establishes a new state-of-the-art, and outperforms prior work by $10.9 \%$ $I o U_{.5}$. Furthermore, we find that some of the top-performing models [8, 35] drew a lot of inspiration from the Bi-directional retrieval model [44]. We conclude that when using strong visual and textual feature representations, this simple model can outperform more complex schemes like MAC [17] and MSRR 9.

Extra Test To verify the validity of the predictions on the leader board, we evaluated the models on an additional hidden test set of 100 commands after the challenge (see $I o U_{.5} \dagger$ in Table 2). None of the submissions experiences a significant performance drop compared to the results on the official test set (see $I o U_{.5}$ vs $I o U_{.5} \dagger$ ). This confirms the validity of the models. The additional test set annotations will be released after the workshop.

Resource Analysis Although the challenge focuses on the quality of the predictions, the used amount of computational resources needs careful consideration too. We performed a detailed resource analysis in Table 2 Except for ASSMR [29], models that improve over MSRR 9] do this at the cost of increasing the model size (parameters). However, we do see improvement in terms of inference speed. The advantage of using a simple bi-directional retrieval approach over a more complex multi-step reasoning process is clearly visible here.

\subsection{Talk2Car Subsets}

Section 3.1 described the construction of four carefully selected smaller test sets on the Talk2Car benchmark. In this section, we evaluate the models under various challenging conditions using the different subsets. Interestingly, the level of difficulty can be tuned as well. As a concrete example, the first subset is constructed by selecting the top- $k$ examples from the dataset for which the object of interest is furthest away. By reducing the value of $k$, we effectively test on objects that are harder to spot due to their increased distance from the 


\begin{tabular}{l|c|c|c|c} 
Model & $I o U_{.5}$ & $I o U_{.5} \dagger$ & Params $(\mathrm{M})$ & Inference Speed $(\mathrm{ms})$ \\
\hline Stacked VLBert [8] & $\mathbf{0 . 7 1 0}$ & $\mathbf{0 . 7 6 2}$ & 683.80 & 320.79 \\
CMRT [25] & 0.691 & 0.713 & 194.97 & 215.50 \\
CMSVG [35] & 0.686 & 0.733 & 366.50 & 164.44 \\
ASSMR [29] & 0.660 & 0.723 & $\mathbf{4 8 . 9 1}$ & 126.23 \\
AttnGrounder [28] & 0.633 & 0.613 & 75.84 & $\mathbf{2 5 . 5 0}$ \\
\hline MSRR [9] & 0.601 & 0.634 & 62.25 & 270.50 \\
MAC [18] & 0.505 & 0.525 & 41.59 & 51.23 \\
Bi-Directional retr. 44] & 0.441 & 0.327 & 15.80 & 100.24
\end{tabular}

Table 2: Results on the Talk2Car test set. The inference speed was measured on a single Nvidia RTX Titan. † Results on an extra smaller test set that was hidden from the leaderboard.

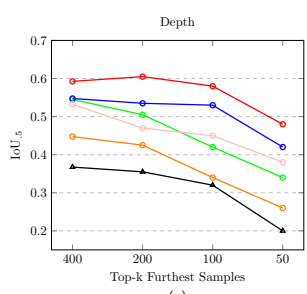

(a)

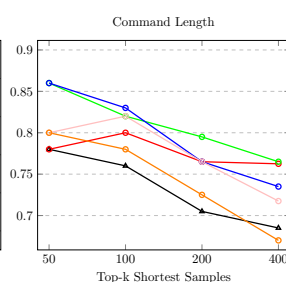

(b)

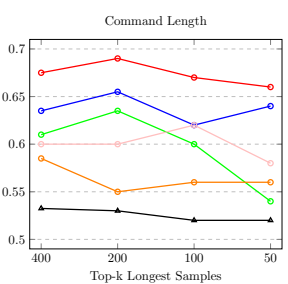

(c)

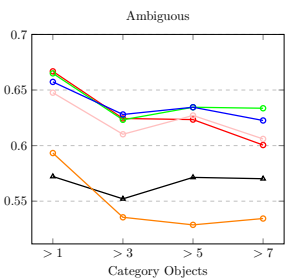

(d)

$\neg$ MSRR $\rightarrow$ - Stacked VLBERT $\rightarrow$ CMRT $\rightarrow$ CMSVG $-\infty$ ASSMR $\rightarrow-$ AttnGrounder

Fig. 3: Results of the state-of-the-art models (Section 3.3 on four sub-test sets from the Talk2Car dataset. Each plot shows the easy examples on the left, while the difficulty increases as we move to the right along the axis. In plot (a) - (c), we increase the difficulty by choosing the top- $k$ samples under the selected criterion (e.g. depth, expression length). In plot (d), we increase the difficulty by choosing samples for which multiple same-category objects are present in the scene.

vehicle. For each subset, we consider four levels of difficulty. Figure 3 shows the results on the subsets. Next, we consider each of the four subsets in detail.

Far Away Objects Figure 3(a) shows the results when focusing on far away objects. All models follow a similar trend, i.e. the performance drops significantly for objects that are further away. This observation follows from the use of the CenterNet [55] region proposals by all models. Notice that far away objects often tend to be small in size. The detection of small objects has been studied by several works, and requires specific dedicated solutions [5, 23].

Short commands Figure 3(b) displays the results when increasing the length of the commands. All models tend to score higher on the commands that are shortest in length. Yet, the performance drops significantly when increasing the length of the commands, i.e. going from the top-50 to the top-400 shortest commands. This is surprising since the maximum sentence length only sees a small 
increase, i.e. from 4 (top-50) to 6 (top-400). We believe that it would be useful to study the reason behind this behavior in future work.

Long commands Figure 3(c) measures the performance on the image-sentence pairs with the top- $k$ longest commands. Depending on the used model, the performance is more susceptible to commands of increasing length. In particular, CMRT 25] shows a decline in performance, while the performance of the other models remains more or less constant (less than $1.5 \%$ difference). We hypothesize that the transformer model [25] responsible for aggregating the image and sentence features does not perform well in combination with long sentences.

Ambiguity Finally, Figure 3(d) shows the performance when considering scenes with an increasing number of objects of the same category as the referred object. The CMRT 25] model obtains the highest performance when considering scenes with many ambiguous objects $(>7)$. In this case, the object of interest can only be identified through its spatial relationship with other objects in the scene. In this case, the global image representation used by the CMRT model is beneficial. Similarly, the MSRR model 9] handles the ambiguous scenes rather well. This can be attributed to its multi-step reasoning process, taking into account all objects and their spatial relationships. The AttnGrounder [28, on the other hand, experiences a significant performance drop on ambiguous cases, potentially due to the fact that it does not utilize region proposals. Appendix A.1 shows a successful case where successive reasoning steps are beneficial.

\subsection{Qualitative comparison}

Finally, Table 3 gives a qualitative overview of all the methods under consideration. In particular, we consider the following elements: model performance, visual backbone, language model, word attention, image augmentations, language augmentations, region proposal based vs non-region proposal based and whether a global image representation is used or not. Based on this comparison, we present some additional findings below.

Region Proposal Networks It is worth noting that all the top-performing models, except for the AttnGrounder [28, are based on pre-computed region proposals. We hypothesize that doing so is particularly interesting under the Talk2Car setting since the scenes are often cluttered with information. This is corroborated by the fact that the models that utilize the region proposals perform better on samples with a large number of objects from the target object category (which often implies a large number of objects in general), as can be seen on Figure 3 . However, there is a downside to the use of region proposals as well. For example, the model can not recover when the region proposal network fails to return a bounding box for the object of interest. In this case, relative regression or query-based approaches can be used [7, 22.

Backbones and word attention Surprisingly, some of the higher ranked methods use the same visual backbone as their lower ranked competitors. On the other hand, higher ranked models can be associated with more recent, better performing language embeddings. We conclude that the effect of using a deeper or better image encoder is smaller compared to using a better linguistic representation. 
Furthermore, word attention seems to be an additional important contributing factor in state-of-the-art models, as it allows them to focus on key words.

Augmentations Remarkably, some models do not use augmentations although it has been shown that these are important for image recognition tasks [43, 38. It is also not surprising that the use of augmentations on the language side has not really been explored, as it is rather difficult. We are interested to see if recent frameworks like [26] can prove helpful in this case.

Global Image Representation Finally, we consider whether a global image representation is taken into account or not. Note that this is optional in case of region proposal based methods. Apart from the Bi-directional retrieval baseline and CMSVG [35, all models used global context information to make the predictions. This observation confirms that it is beneficial to use global image information. Doing so allows to better capture the spatial relationships between objects. These are likely important to tackle the C4AV challenge.

\section{Conclusion}

In this paper, we reviewed the results of the Commands for Autonomous Vehicles challenge held at ECCV20'. First, we presented an overview of various strategies to tackle the visual grounding task. For each method, we described its key components, and discussed the commonalities and differences with existing works. Second, we presented an extensive experimental evaluation of the considered methods. We briefly discuss some of the limitations and possibilities for future work.

Dataset The Talk2Car dataset provides a more realistic task setting compared to existing benchmarks [21, 54, 27, yet we identify several possibilities to extend this work. First, the dataset could be further up-scaled in terms of the number of commands, and the variety of the environments. Second, it would be interesting to add annotations containing novel classes [36] and groups of objects.

Extra Modalities Surprisingly, the use of other sensor modalities, e.g. depth, LIDAR, maps, etc., remains unexplored. Still, this provides an interesting direction for future research. In particular, leveraging additional data sources as extra input [1] or auxiliary task [45] is expected to boost the performance.

Navigation The current setup considers the task of visual grounding in isolation. Yet, the agent is also responsible for navigating to the correct destination. Extending the current setup with a navigation task [2, 37, 46, 42, 6] would provide a useful addition.

\section{Acknowledgements}

This project is sponsored by the MACCHINA project from the KU Leuven with grant number C14/18/065. Additionally, we acknowledge support by the Flemish Government under the Artificial Intelligence (AI) Flanders programme. Finally, we thank Huawei for sponsoring the workshop and AICrowd for hosting our challenge. 
Deruyttere T., Vandenhende S., Grujicic D. et al.

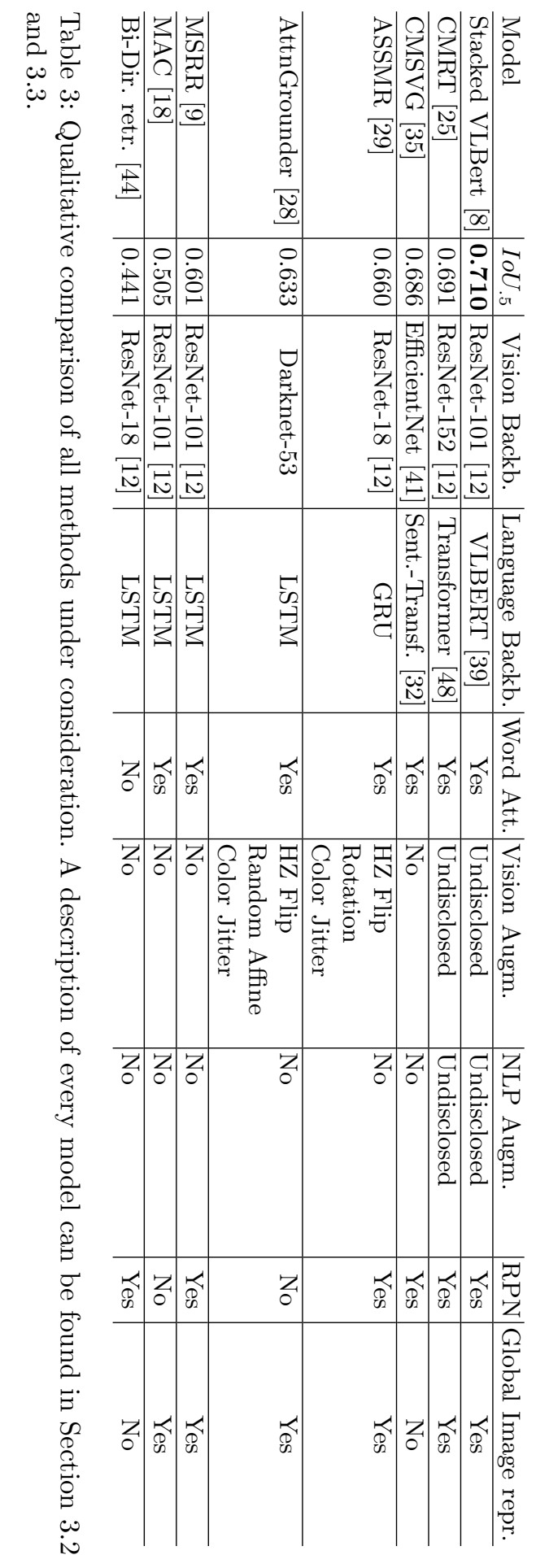




\section{Bibliography}

[1] Anderson, P., He, X., Buehler, C., Teney, D., Johnson, M., Gould, S., Zhang, L.: Bottom-up and top-down attention for image captioning and visual question answering. In: Proceedings of the IEEE conference on computer vision and pattern recognition. pp. 6077-6086 (2018)

[2] Anderson, P., Wu, Q., Teney, D., Bruce, J., Johnson, M., Sünderhauf, N., Reid, I., Gould, S., van den Hengel, A.: Vision-and-language navigation: Interpreting visually-grounded navigation instructions in real environments. In: Proceedings of the IEEE Conference on Computer Vision and Pattern Recognition. pp. 3674-3683 (2018)

[3] Antol, S., Agrawal, A., Lu, J., Mitchell, M., Batra, D., Lawrence Zitnick, C., Parikh, D.: Vqa: Visual question answering. In: Proceedings of the IEEE international conference on computer vision. pp. 2425-2433 (2015)

[4] Caesar, H., Bankiti, V., Lang, A.H., Vora, S., Liong, V.E., Xu, Q., Krishnan, A., Pan, Y., Baldan, G., Beijbom, O.: nuscenes: A multimodal dataset for autonomous driving. In: Proceedings of the IEEE/CVF Conference on Computer Vision and Pattern Recognition. pp. 11621-11631 (2020)

[5] Chen, C., Liu, M.Y., Tuzel, O., Xiao, J.: R-cnn for small object detection. In: Asian conference on computer vision. pp. 214-230. Springer (2016)

[6] Chen, H., Suhr, A., Misra, D., Snavely, N., Artzi, Y.: Touchdown: Natural language navigation and spatial reasoning in visual street environments. In: Proceedings of the IEEE Conference on Computer Vision and Pattern Recognition. pp. 12538-12547 (2019)

[7] Chen, K., Kovvuri, R., Nevatia, R.: Query-guided regression network with context policy for phrase grounding. In: The IEEE International Conference on Computer Vision (ICCV) (2017)

[8] Dai, H., Luo, S., Ding, Y., Shao, L.: Commands for autonomous vehicles by progressively stacking visual-linguistic representations. In: Proceedings of the 16th European Conference on Computer Vision, 2020. Commands for Autonomous Vehicles (C4AV) ECCV Workshop (2020)

[9] Deruyttere, T., Collell, G., Moens, M.F.: Giving commands to a self-driving car: A multimodal reasoner for visual grounding. In: Reasoning for Complex QA Workshop, AAAI (2020)

[10] Deruyttere, T., Vandenhende, S., Grujicic, D., Van Gool, L., Moens, M.F.: Talk2car: Taking control of your self-driving car. In: Proceedings of the 2019 Conference on Empirical Methods in Natural Language Processing and the 9th International Joint Conference on Natural Language Processing (EMNLP-IJCNLP). pp. 2088-2098 (2019)

[11] Gupta, S., Girshick, R., Arbeláez, P., Malik, J.: Learning rich features from rgb-d images for object detection and segmentation. In: European conference on computer vision. pp. 345-360. Springer (2014)

[12] He, K., Zhang, X., Ren, S., Sun, J.: Deep residual learning for image recognition. CoRR abs/1512.03385 (2015), http://arxiv.org/abs/1512.03385 
[13] Hodosh, M., Young, P., Hockenmaier, J.: Framing image description as a ranking task: Data, models and evaluation metrics. Journal of Artificial Intelligence Research 47, 853-899 (2013)

[14] Hu, R., Andreas, J., Darrell, T., Saenko, K.: Explainable neural computation via stack neural module networks. CoRR abs/1807.08556 (2018), http://arxiv.org/abs/1807.08556

[15] Hu, R., Andreas, J., Darrell, T., Saenko, K.: Explainable neural computation via stack neural module networks. In: Proceedings of the European conference on computer vision (ECCV). pp. 53-69 (2018)

[16] Hu, R., Xu, H., Rohrbach, M., Feng, J., Saenko, K., Darrell, T.: Natural language object retrieval. In: Proceedings of the IEEE Conference on Computer Vision and Pattern Recognition. pp. 4555-4564 (2016)

[17] Hudson, D.A., Manning, C.D.: Compositional attention networks for machine reasoning. CoRR abs/1803.03067 (2018), http://arxiv.org/abs/ 1803.03067

[18] Hudson, D.A., Manning, C.D.: Compositional attention networks for machine reasoning (2018)

[19] Johnson, J., Hariharan, B., Maaten, L.V.D., Hoffman, J., Fei-Fei, L., Zitnick, C.L., Girshick, R.: Inferring and Executing Programs for Visual Reasoning. In: Proceedings of the IEEE International Conference on Computer Vision. vol. 2017-Octob, pp. 3008-3017 (2017)

[20] Karpathy, A., Joulin, A., Fei-Fei, L.F.: Deep fragment embeddings for bidirectional image sentence mapping. In: Advances in neural information processing systems. pp. 1889-1897 (2014)

[21] Kazemzadeh, S., Ordonez, V., Matten, M., Berg, T.: Referitgame: Referring to objects in photographs of natural scenes. In: Proceedings of the 2014 Conference on Empirical Methods in Natural Language Processing (EMNLP). pp. 787-798 (2014)

[22] Kovvuri, R., Nevatia, R.: Pirc net: Using proposal indexing, relationships and context for phrase grounding. In: Asian Conference on Computer Vision. pp. 451-467. Springer (2018)

[23] Li, J., Liang, X., Wei, Y., Xu, T., Feng, J., Yan, S.: Perceptual generative adversarial networks for small object detection. In: Proceedings of the IEEE conference on computer vision and pattern recognition. pp. 1222-1230 (2017)

[24] Lin, T., Maire, M., Belongie, S.J., Bourdev, L.D., Girshick, R.B., Hays, J., Perona, P., Ramanan, D., Dollár, P., Zitnick, C.L.: Microsoft COCO: common objects in context. CoRR abs/1405.0312 (2014), http://arxiv. org/abs/1405.0312

[25] Luo, S., Dai, H., Shao, L., Ding, Y.: Cross-modal representations from transformer. In: Proceedings of the 16th European Conference on Computer Vision, 2020. Commands for Autonomous Vehicles (C4AV) Workshop (2020)

[26] Ma, E.: Nlp augmentation. https://github.com/makcedward/nlpaug (2019)

[27] Mao, J., Huang, J., Toshev, A., Camburu, O., Yuille, A.L., Murphy, K.: Generation and comprehension of unambiguous object descriptions. In: Proceedings of the IEEE conference on computer vision and pattern recognition. pp. 11-20 (2016) 
[28] Mittal, V.: Attngrounder: Talking to cars with attention. In: Proceedings of the 16th European Conference on Computer Vision, 2020. Commands for Autonomous Vehicles (C4AV) Workshop (2020)

[29] Ou, J., Zhang, X.: Attention enhanced single stage multi-modal reasoner. In: Proceedings of the 16th European Conference on Computer Vision, 2020. Commands for Autonomous Vehicles (C4AV) ECCV Workshop (2020)

[30] Paszke, A., Gross, S., Massa, F., Lerer, A., Bradbury, J., Chanan, G., Killeen, T., Lin, Z., Gimelshein, N., Antiga, L., et al.: Pytorch: An imperative style, high-performance deep learning library. In: Advances in neural information processing systems. pp. 8026-8037 (2019)

[31] Redmon, J., Farhadi, A.: Yolov3: An incremental improvement. arXiv preprint arXiv:1804.02767 (2018)

[32] Reimers, N., Gurevych, I.: Sentence-bert: Sentence embeddings using siamese bert-networks. In: Proceedings of the 2019 Conference on Empirical Methods in Natural Language Processing. Association for Computational Linguistics (11 2019), http://arxiv.org/abs/1908.10084

[33] Ren, S., He, K., Girshick, R.B., Sun, J.: Faster R-CNN: towards real-time object detection with region proposal networks. CoRR abs/1506.01497 (2015), http://arxiv.org/abs/1506.01497

[34] Rohrbach, A., Rohrbach, M., Hu, R., Darrell, T., Schiele, B.: Grounding of textual phrases in images by reconstruction. In: European Conference on Computer Vision. pp. 817-834. Springer (2016)

[35] Rufus, N., Nair, U., Krishnam, M., Gandhi, V.: Cosine meets softmax: A tough-to-beat baseline for visual grounding. In: Proceedings of the 16th European Conference on Computer Vision, 2020. Commands for Autonomous Vehicles (C4AV) Workshop (2020)

[36] Sadhu, A., Chen, K., Nevatia, R.: Zero-shot grounding of objects from natural language queries (2019)

[37] Savva, M., Kadian, A., Maksymets, O., Zhao, Y., Wijmans, E., Jain, B., Straub, J., Liu, J., Koltun, V., Malik, J., et al.: Habitat: A platform for embodied ai research. In: Proceedings of the IEEE International Conference on Computer Vision. pp. 9339-9347 (2019)

[38] Sohn, K., Berthelot, D., Li, C.L., Zhang, Z., Carlini, N., Cubuk, E.D., Kurakin, A., Zhang, H., Raffel, C.: Fixmatch: Simplifying semi-supervised learning with consistency and confidence (2020)

[39] Su, W., Zhu, X., Cao, Y., Li, B., Lu, L., Wei, F., Dai, J.: Vl-bert: Pretraining of generic visual-linguistic representations. In: International Conference on Learning Representations (2020), https://openreview.net/ forum?id=SygXPaEYvH

[40] Suarez, J., Johnson, J., Li, F.F.: DDRprog: A CLEVR Differentiable Dynamic Reasoning Programmer (2018), http://arxiv.org/abs/1803. 11361

[41] Tan, M., Le, Q.V.: Efficientnet: Rethinking model scaling for convolutional neural networks. arXiv preprint arXiv:1905.11946 (2019)

[42] Thomason, J., Murray, M., Cakmak, M., Zettlemoyer, L.: Vision-and-dialog navigation. In: Conference on Robot Learning. pp. 394-406 (2020) 
[43] Van Gansbeke, W., Vandenhende, S., Georgoulis, S., Proesmans, M., Van Gool, L.: Scan: Learning to classify images without labels. In: European Conference on Computer Vision (ECCV) (2020)

[44] Vandenhende, S., Deruyttere, T., Grujicic, D.: A baseline for the commands for autonomous vehicles challenge. arXiv preprint arXiv:2004.13822 (2020)

[45] Vandenhende, S., Georgoulis, S., Proesmans, M., Dai, D., Van Gool, L.: Revisiting multi-task learning in the deep learning era. arXiv preprint arXiv:2004.13379 (2020)

[46] Vasudevan, A.B., Dai, D., Van Gool, L.: Talk2nav: Long-range vision-andlanguage navigation in cities. arXiv preprint arXiv:1910.02029 (2019)

[47] Vasudevan, A.B., Dai, D., Van Gool, L., Zurich, E.: Object referring in videos with language and human gaze (2018)

[48] Vaswani, A., Shazeer, N., Parmar, N., Uszkoreit, J., Jones, L., Gomez, A., Kaiser, L., Polosukhin, I.: Attention is all you need. arxiv 2017. arXiv preprint arXiv:1706.03762 (2017)

[49] Vinyals, O., Toshev, A., Bengio, S., Erhan, D.: Show and tell: A neural image caption generator. In: Proceedings of the IEEE conference on computer vision and pattern recognition. pp. 3156-3164 (2015)

[50] Wang, L., Li, Y., Huang, J., Lazebnik, S.: Learning two-branch neural networks for image-text matching tasks. IEEE Transactions on Pattern Analysis and Machine Intelligence 41(2), 394-407 (2018)

[51] Xu, K., Ba, J., Kiros, R., Cho, K., Courville, A., Salakhudinov, R., Zemel, R., Bengio, Y.: Show, attend and tell: Neural image caption generation with visual attention. In: International conference on machine learning. pp. 2048-2057 (2015)

[52] Young, P., Lai, A., Hodosh, M., Hockenmaier, J.: From image descriptions to visual denotations: New similarity metrics for semantic inference over event descriptions. Transactions of the Association for Computational Linguistics 2, 67-78 (2014)

[53] Yu, L., Lin, Z., Shen, X., Yang, J., Lu, X., Bansal, M., Berg, T.L.: Mattnet: Modular attention network for referring expression comprehension. In: Proceedings of the IEEE Conference on Computer Vision and Pattern Recognition. pp. 1307-1315 (2018)

[54] Yu, L., Poirson, P., Yang, S., Berg, A.C., Berg, T.L.: Modeling context in referring expressions. In: European Conference on Computer Vision. pp. 69-85. Springer (2016)

[55] Zhou, X., Wang, D., Krähenbühl, P.: Objects as points. arXiv preprint arXiv:1904.07850 (2019) 


\section{Appendix A}

\section{A.1 Multi-step Reasoning MSRR}

This section discusses the influence of having reasoning steps and showcases an example where the MSRR 9] successfully finds the correct answer for the command by using multiple reasoning steps.

First, we will look at the influence of reasoning steps. Assume we have a MSRR model that uses 10 reasoning steps, Figure 4 shows in which of these 10 reasoning steps the model makes its final prediction. It is clear that most of the final predictions are made in the very first reasoning step. For instance, if we would only consider the answers in the first step and ignore any change of decision in the following steps, we would achieve $\approx 55 \% I o U .5$. Yet, by including more reasoning steps we can further improve this to $\approx 60 \% I_{0} U_{.5}$. This shows that having reasoning steps can be beneficial for this kind of task.

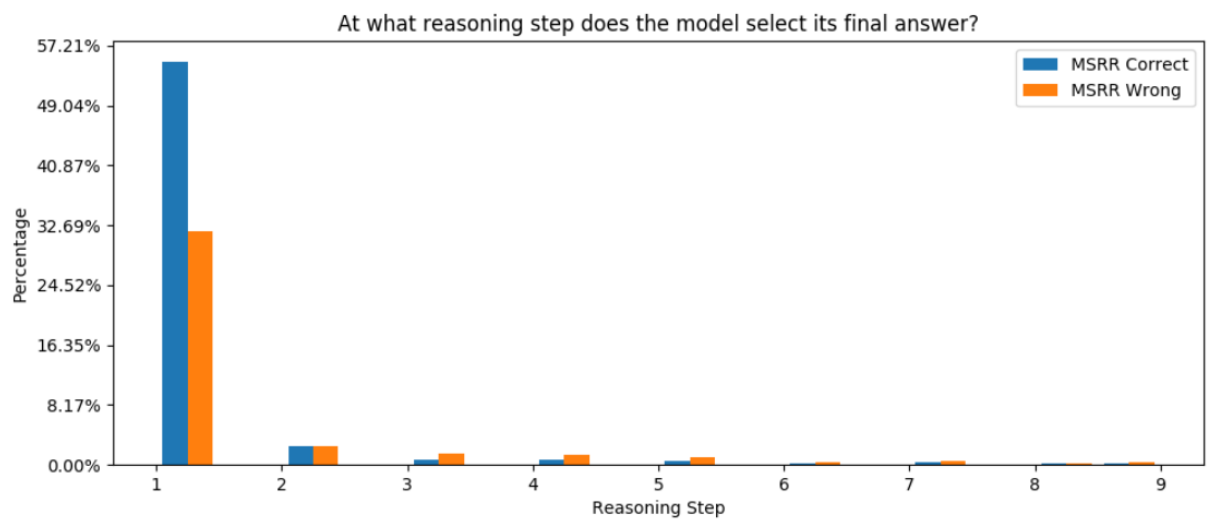

Fig. 4: This plot shows in which step a 10 reasoning step MSRR makes its final decision. We use MSRR Correct (blue) to indicate when the final answer by the model is also the correct answer while MSRR Wrong (orange) is used when the final answer is the wrong answer.

The example used in this section uses a specific visualisation that first needs to be introduced. In the Figures 5 and 6 , we explain in detail this visualisation. Then, Figure 7 shows the starting state of the MSRR. Figure 8 shows that the model makes a wrong decission at first but in Figure 9, and after six reasoning steps, we see that the model selects the correct answer. Finally, in Figure 10, we see that the object selected after six reasoning step, is the final output of the model. 


\section{Figure explanation (1)}

- The coloured bounding boxes are the predicted bounding boxes from CenterNet

- The green filled box is the ground truth referred object. The big green arrow also points to the referred object

\section{- Under the image we can find the command}

- Under the command we can find the regions (with coloured borders to indicate which bounding box in the image is being used) together with their scores during the reasoning process

- The scores indicated with a green box, are the scores of the correct answer

- The scores indicated in bold represent the region that currently has the highest score. This is also indicated in the image with a bigger border around the object

- Finally, next to each score there is a $\uparrow, \downarrow$ or $=$ to indicate if the score has increased, decreased or remained the same respectively compared to the previous reasoning step

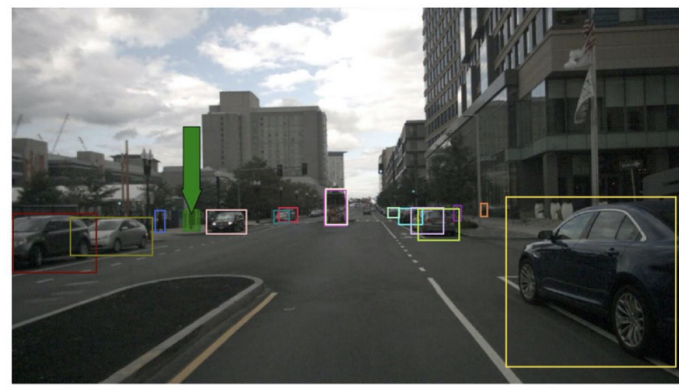

Command: $i$ think that is jim over there next to that parked black car on the other side. stop when parallel to him.

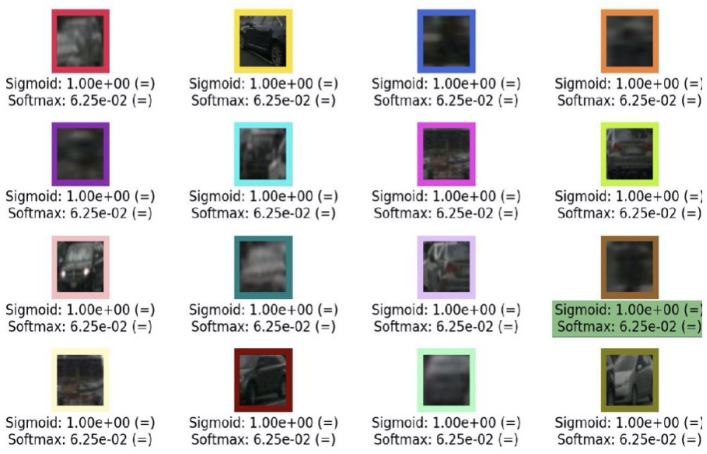

Fig. 5: Explaining the visualisation of the reasoning process (Part 1). Figure from [9]. 


\section{Figure explanation (2)}

- During the reasoning process, the words that are being focussed on are marked with a red background where the alpha channel corresponds with their attention weight. $=>$ Bright red background means high attention on that word, light red means low attention

- The object with the highest score will be indicated (i) with an arrow in the image, (ii) the other regions in the image will become less visible and (iii) in the plot under the command, the other regions will also be less visible

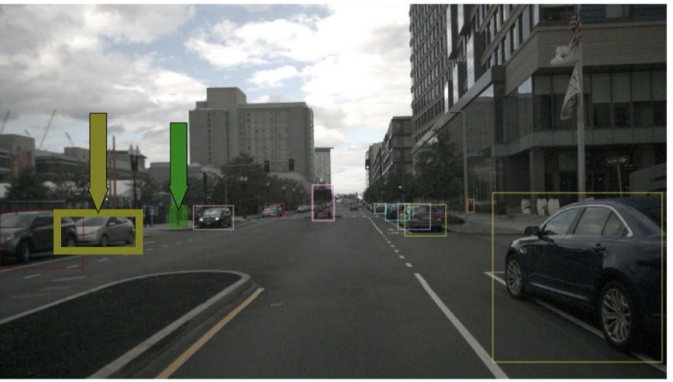

Command: $i$ think that is jim over there next to that parked black car on the other side. stop when parallel to him.

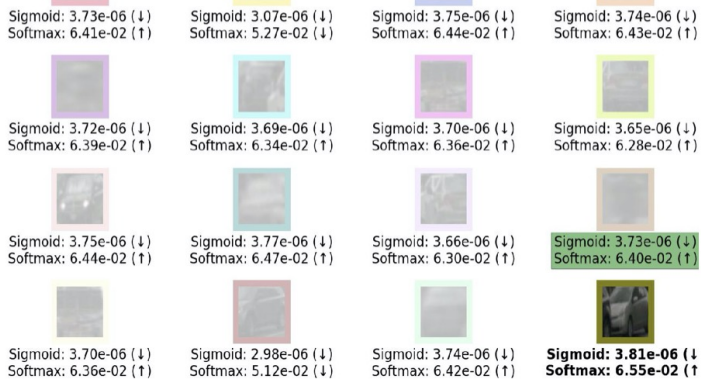

Fig. 6: Explaining the visualisation of the reasoning process (Part 2). Figure from [9]. 


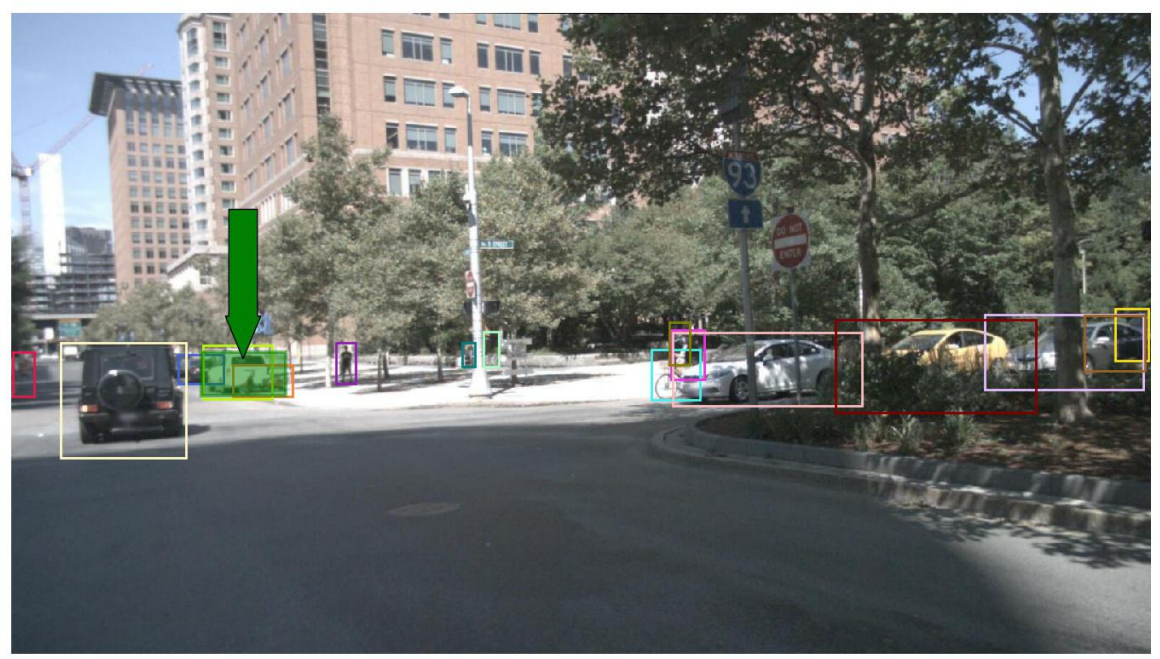

\section{Command: find me a parking spot near that white car up ahead. not the one on the right.}

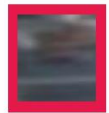

Sigmoid: $1.00 \mathrm{e}+00(=)$ Softmax: $6.25 \mathrm{e}-02(=)$

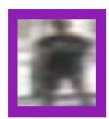

Sigmoid: $1.00 \mathrm{e}+00(=)$ Softmax: 6.25e-02 (=)

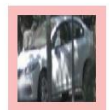

Sigmoid: $1.00 \mathrm{e}+00(=)$ Softmax: $6.25 \mathrm{e}-02(=)$

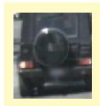

Sigmoid: $1.00 \mathrm{e}+00(=)$ Softmax: $6.25 \mathrm{e}-02(=)$

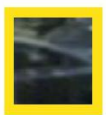

Sigmoid: $1.00 \mathrm{e}+00(=)$ Softmax: $6.25 \mathrm{e}-02(=)$

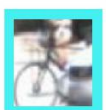

Sigmoid: $1.00 \mathrm{e}+00(=)$ Softmax: $6.25 \mathrm{e}-02(=)$

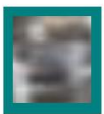

Sigmoid: $1.00 e+00(=)$ Softmax: $6.25 \mathrm{e}-02(=)$

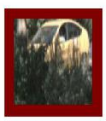

Sigmoid: $1.00 e+00(=)$ Softmax: $6.25 \mathrm{e}-02(=)$

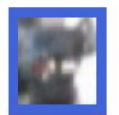

Sigmoid: $1.00 \mathrm{e}+00(=)$ Softmax: $6.25 \mathrm{e}-02(=)$

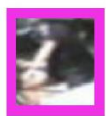

Sigmoid: $1.00 \mathrm{e}+00(=)$ Softmax: $6.25 \mathrm{e}-02(=)$

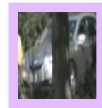

Sigmoid: $1.00 \mathrm{e}+00(=)$ Softmax: $6.25 \mathrm{e}-02(=)$

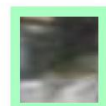

Sigmoid: $1.00 \mathrm{e}+00(=)$ Softmax: $6.25 \mathrm{e}-02(=)$

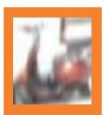

Sigmoid: $1.00 e+00(=)$ Softmax: $6.25 \mathrm{e}-02(=)$

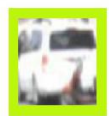

Sigmoid: $1.00 e+00(=)$ Softmax: $6.25 \mathrm{e}-02(=)$

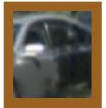

Sigmoid: $1.00 \mathrm{e}+00(=)$ Softmax: $6.25 \mathrm{e}-02(=)$

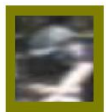

Sigmoid: $1.00 \mathrm{e}+00(=)$ Softmax: $6.25 \mathrm{e}-02(=)$

Fig. 7: Example 3 - The state of the model before the reasoning process starts for the given command, regions and image. Figure from [9]. 


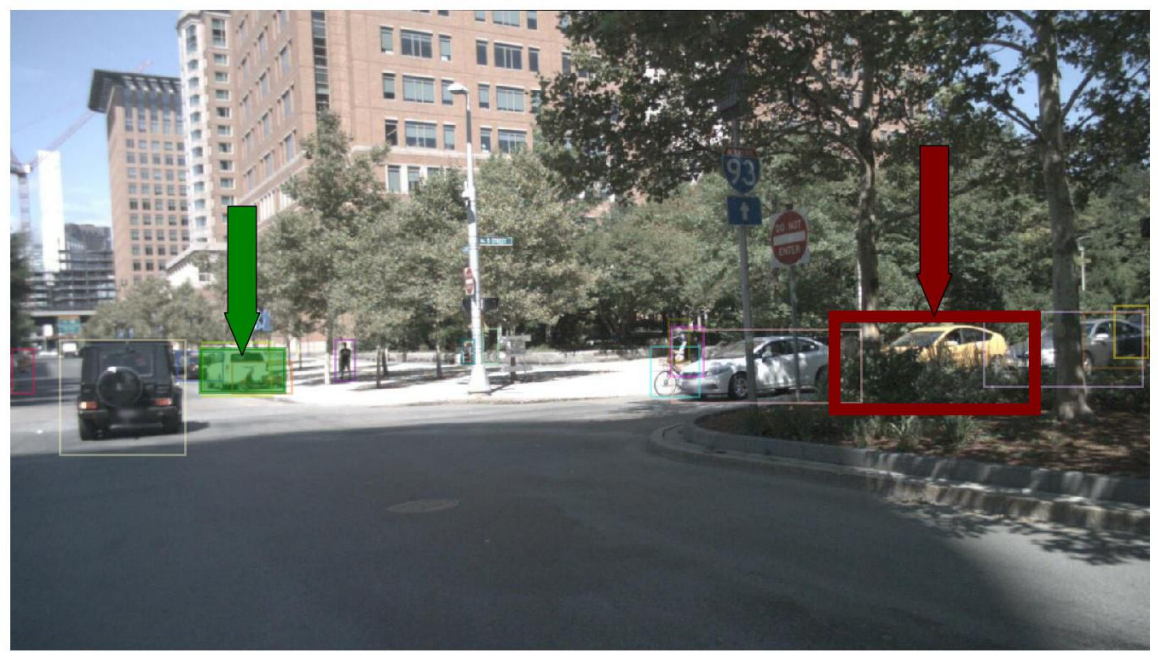

\section{Command: find me a parking spot near that white car up ahead. not the one on the right.}



Sigmoid: $9.75 \mathrm{e}-06(\downarrow)$ Softmax: $6.32 \mathrm{e}-02(\uparrow)$

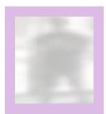

Sigmoid: $9.75 \mathrm{e}-06(\downarrow)$ Softmax: $6.31 \mathrm{e}-02(\uparrow)$

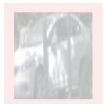

Sigmoid: $9.52 \mathrm{e}-06(\downarrow)$ Softmax: $6.17 \mathrm{e}-02(\downarrow)$

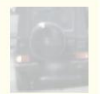

Sigmoid: $9.90 \mathrm{e}-06(\downarrow)$ Softmax: $6.41 \mathrm{e}-02(\uparrow)$

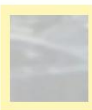

Sigmoid: $9.72 \mathrm{e}-06(\downarrow)$ Softmax: $6.29 \mathrm{e}-02(\uparrow)$

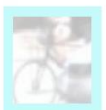

Sigmoid: $9.33 \mathrm{e}-06(\downarrow)$ Softmax: $6.04 \mathrm{e}-02(\downarrow)$

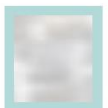

Sigmoid: $9.75 \mathrm{e}-06(\downarrow)$ Softmax: $6.32 \mathrm{e}-02(\uparrow)$

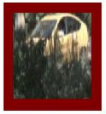

Sigmoid: 1.03e-05 ( $\downarrow$ ) Softmax: 6.65e-02 $(\uparrow)$

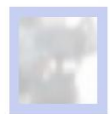

Sigmoid: $9.93 \mathrm{e}-06(\downarrow)$ Softmax: $6.43 e-02(\uparrow)$

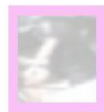

Sigmoid: 9.64e-06 ( $\downarrow)$ Softmax: $6.24 \mathrm{e}-02(\downarrow)$

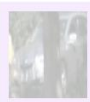

Sigmoid: $9.80 \mathrm{e}-06(\downarrow)$ Softmax: $6.35 \mathrm{e}-02(\uparrow)$

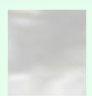

Sigmoid: $9.75 \mathrm{e}-06(\downarrow)$ Softmax: 6.32e-02 $(\uparrow)$
Sigmoid: 9.32e-06 ( $\downarrow)$

Softmax: $6.04 \mathrm{e}-02(\downarrow)$

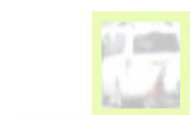

Sigmoid: $8.59 \mathrm{e}-06(\downarrow)$ Softmax: $5.57 \mathrm{e}-02(\downarrow)$

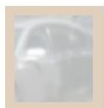

Sigmoid: $9.72 \mathrm{e}-06(\downarrow)$ Softmax: $6.29 \mathrm{e}-02(\uparrow)$

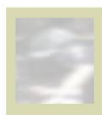

Sigmoid: $9.66 \mathrm{e}-06(\downarrow)$ Softmax: $6.26 \mathrm{e}-02(\uparrow)$

Fig. 8: Example 3 - Visualization of reasoning process. Step 1. Figure from [9]. 


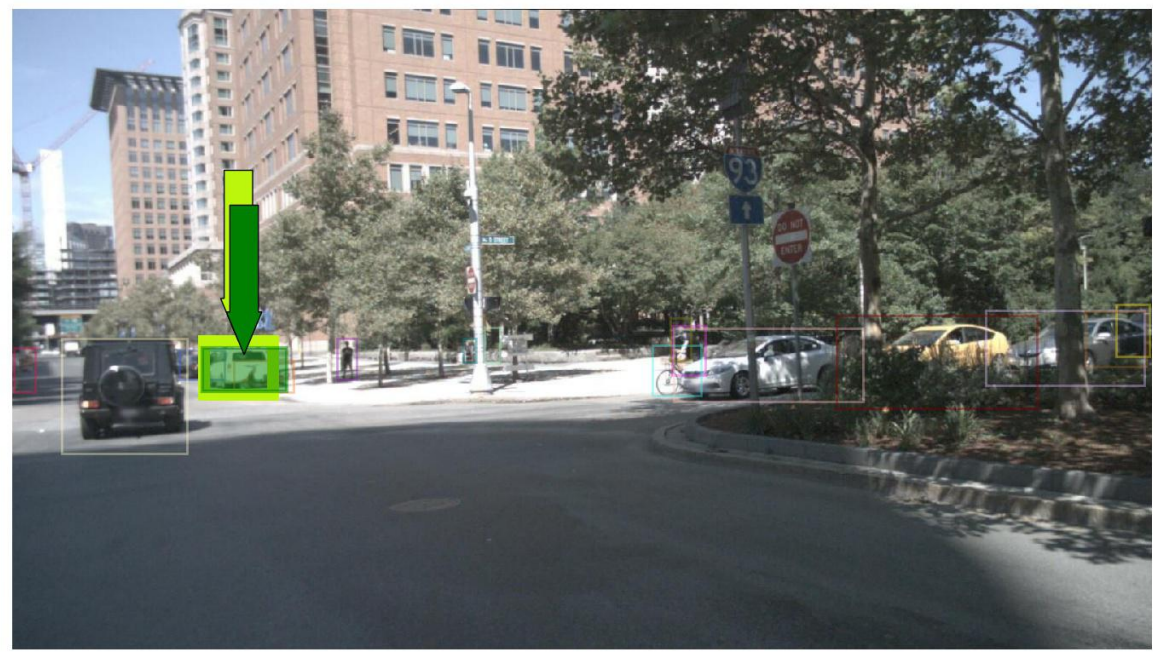

\section{Command: find me a parking spot near that white car up ahead. not the one on the right.}

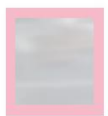

Sigmoid: $1.17 \mathrm{e}-11(\downarrow)$ Softmax: 6.18e-02 ( $\downarrow)$

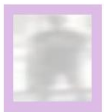

Sigmoid: $1.17 \mathrm{e}-11(\downarrow)$ Softmax: 6.18e-02 ( $\downarrow)$

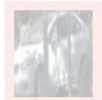

Sigmoid: $1.14 \mathrm{e}-11(\downarrow)$ Softmax: 6.04e-02 $(\downarrow)$

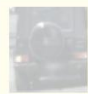

Sigmoid: 1.16e-11 ( $\downarrow)$ Softmax: $6.14 \mathrm{e}-02(\downarrow)$

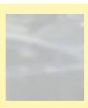

Sigmoid: $1.17 \mathrm{e}-11(\downarrow)$ Softmax: $6.19 \mathrm{e}-02(\downarrow)$

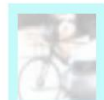

Sigmoid: $1.20 \mathrm{e}-11(\downarrow)$ Softmax: $6.37 \mathrm{e}-02(\uparrow)$

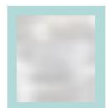

Sigmoid: $1.17 \mathrm{e}-11$ ( $\downarrow)$ Softmax: $6.18 \mathrm{e}-02(\downarrow)$

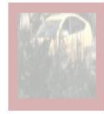

Sigmoid: $1.08 \mathrm{e}-11(\downarrow)$ Softmax: $5.71 \mathrm{e}-02(\downarrow)$

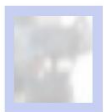

Sigmoid: $1.19 \mathrm{e}-11(\downarrow)$ Softmax: $6.29 \mathrm{e}-02(\uparrow)$

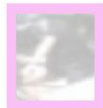

Sigmoid: $1.18 \mathrm{e}-11(\downarrow)$ Softmax: $6.25 \mathrm{e}-02(\downarrow)$

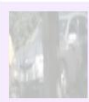

Sigmoid: $1.15 \mathrm{e}-11(\downarrow)$ Softmax: $6.12 \mathrm{e}-02(\downarrow)$

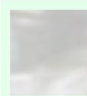

Sigmoid: $1.17 \mathrm{e}-11(\downarrow)$ Softmax: $6.18 \mathrm{e}-02(\downarrow)$

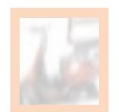

Sigmoid: $1.20 \mathrm{e}-11(\downarrow)$ Softmax: $6.37 \mathrm{e}-02(\uparrow)$

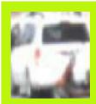

Sigmoid: $1.39 \mathrm{e}-11(\downarrow)$ Softmax: $7.39 \mathrm{e}-02(\uparrow)$

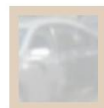

Sigmoid: 1.17e-11 ( $\downarrow)$ Softmax: $6.18 \mathrm{e}-02(\downarrow)$

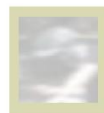

Sigmoid: $1.17 \mathrm{e}-11(\downarrow)$ Softmax: $6.22 \mathrm{e}-02(\downarrow)$

Fig. 9: Example 3 - Visualization of reasoning process. Step 6. Figure from [9]. 


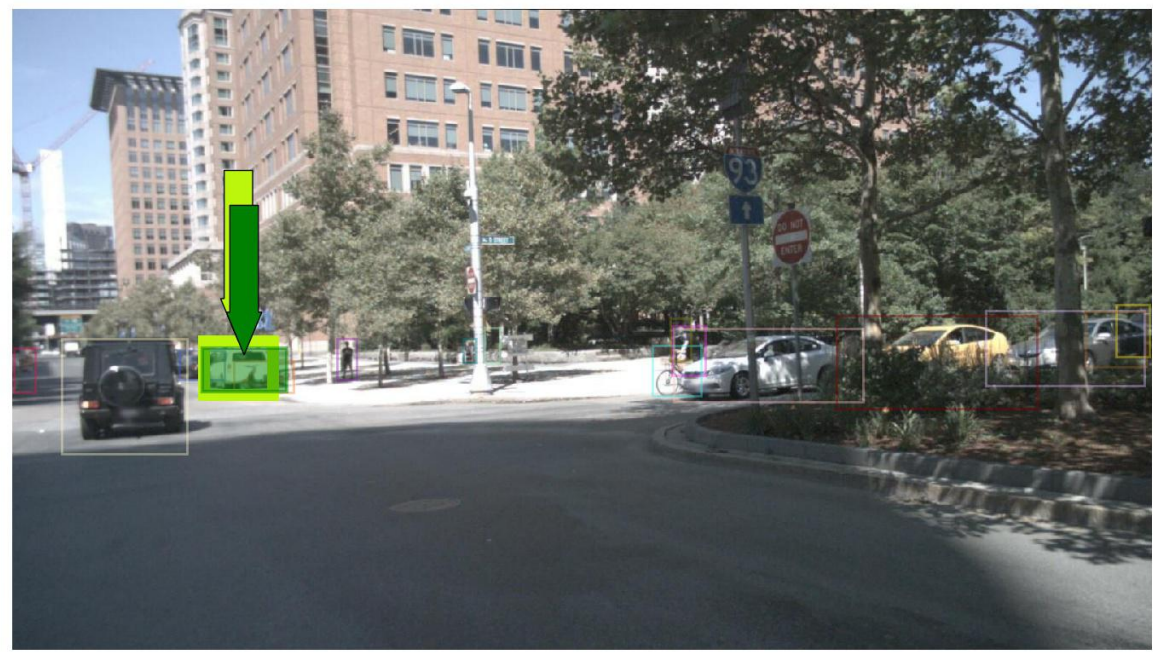

Command: find me a parking spot near that white car up ahead. not the one on the right.

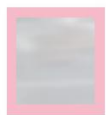

Sigmoid: $3.55 \mathrm{e}-35(\downarrow)$ Softmax: $1.72 \mathrm{e}-06(\downarrow)$

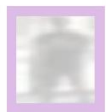

Sigmoid: $3.58 \mathrm{e}-35(\downarrow)$ Softmax: 1.74e-06 $(\downarrow)$

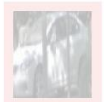

Sigmoid: 6.94e-33 ( $\downarrow)$ Softmax: 3.37e-04 ( $\downarrow)$

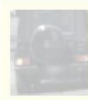

Sigmoid: $4.03 e-35(\downarrow)$ Softmax: $1.96 \mathrm{e}-06(\downarrow)$

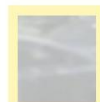

Sigmoid: 4.50e-35 ( $\downarrow)$ Softmax: 2.19e-06 ( $\downarrow)$

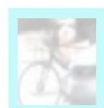

Sigmoid: $2.33 \mathrm{e}-34(\downarrow)$ Softmax: $1.14 \mathrm{e}-05(\downarrow)$

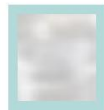

Sigmoid: $3.57 e-35(\downarrow)$ Softmax: $1.73 \mathrm{e}-06(\downarrow)$

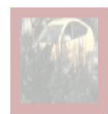

Sigmoid: $3.31 \mathrm{e}-36(\downarrow)$

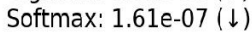

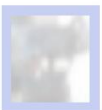

Sigmoid: 8.02e-34 ( $\downarrow$ ) Softmax: $3.90 \mathrm{e}-05(\downarrow)$

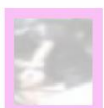

Sigmoid: $6.66 \mathrm{e}-35(\downarrow)$ Softmax: 3.24e-06( $(\downarrow)$

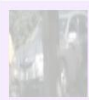

Sigmoid: $2.93 e-35(\downarrow)$ Softmax: $1.43 \mathrm{e}-06(\downarrow)$

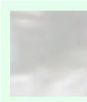

Sigmoid: $3.57 e-35(\downarrow)$ Softmax: $1.74 \mathrm{e}-06(\downarrow)$

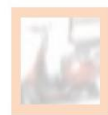

Sigmoid: $2.95 \mathrm{e}-33(\downarrow)$ Softmax: $1.44 \mathrm{e}-04(\downarrow)$

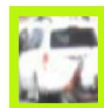

Sigmoid: 2.05e-29 ( $\downarrow$ ) Softmax: $9.99 \mathrm{e}-01(\uparrow)$

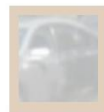

Sigmoid: 4.36e-35 ( $\downarrow)$ Softmax: 2.12e-06 ( $\downarrow)$



Sigmoid: $5.69 \mathrm{e}-35(\downarrow)$ Softmax: $2.77 \mathrm{e}-06(\downarrow)$

Fig. 10: Example 3 - Visualization of reasoning process. Final step. Figure from 9]. 